\title{
Novel Production Methods
}

\section{HOT COMPACTION OF POLYMERIC FIBRES}

The last twenty years have seen the development of high stiffness and high strength polymers by a number of different processing routes. The materials produced range from very high stiffness fibres (up to at least $90 \mathrm{GPa}$ ), manufactured by drawing isotropic fibres to a very high draw ratio, to thick-section products produced by pressing or pulling polymer through a die, whose stiffness is usually limited to $<40 \mathrm{GPa}$. There is therefore a requirement for high stiffness, thick-section materials. A process [British Patent: GB 2253420] for manufacturing such materials has been developed at the IRC in Polymer Science and Technology, Leeds University, UK. Termed "hot compaction", it takes highly drawn fibres and by choosing suitable conditions of temperature and pressure produces a homogeneous product which retains a high proportion of the original fibre properties while achieving a reasonable strength [J. Mater. Sci., to be published]

The important step was the discovery that it was possible to take a fibre below its melting range and selectively melt a small portion. Sufficient pressure needs to be applied to stop the fibre shrinking and losing its orientation, but not enough to inhibit melting. It is obviously an advantage to have the surface of the fibre melt first and anything that promotes this is desirable. For a hexagonal close-packed structure (a unidirectional arrangement of fibres) only $9 \%$ of the second phase is needed to fill all the holes and bind the structure, allowing substantial retention of fibre properties.

On cooling, the molten material recrystallises to form a second, lower melting point phase, clearly evident in differential scanning calorimetry. Morphological studies show the second phase to form a transcrystalline bridge between the fibres. The spaces between the fibres are completely filled with the melted and recrystallised polyethylene. Compaction is seen to be complete, and the fibres are in the main, still largely circular in cross-section.

Nucleation of the second phase is all along the fibre boundaries so that crystals grow out perpendicular to the fibres, meeting in the middle and forming a very strong bond. There is evidence from fracture studies that failure of the compacted composite occurs within the fibre itself, suggesting the melted and recrystallised material forms an excellent "glue", and that the weakness is within the fibre itself. This is confirmed by compaction studies on a range of fibres, which show the most highly aligned fibres (i.e., those with the lowest transverse properties) as having the lowest composite transverse strengths.

Fibres so far successfully compacted include melt spun, melt kneaded and gel-spun polyethylenes, polypropylene and PET. Other fibre configurations can be compacted including chopped fibres, woven fibre cloth and laminates of unidirectional fibres. All of these have improved strength compared to unidirectionally aligned fibres, but have lower stiffness. The compacted composites allow a light, tough and high stiffness composite to be manufactured using a single phase. This has considerable advantages over processing two-component composites.

P.J. Hine and I.M. Ward, IRC in Polymer Science \& Technology, Leeds, UK

R.H. Olley and D.C. Bassett, University of Reading, UK

\section{IBM RESEARCH}

\section{Shifting Emphasis to Remain Vital}

Dr. Karl Kümmerle, Director of the IBM Zurich Research Laboratory, discusses the impact on basic research of the company's "fundamental redefinition" announced last November, and the latest round of staff reductions.

IBM's Zurich Research Laboratory with a staff of about 200 represents a fairly small part of the Research Division (3300 staff and an annual budget of 600 M\$US which has remained almost constant for several years). It is dwarfed by IBM as a whole (300 000 employees; 69000 M\$US turn-over in 1991). Founded in 1956, established on the present site in 1962, expanded in the 1970's, modernised and expanded again in the mid-1980's, the Zurich lab, with its two Nobel prizes for discovering scanning tunnelling microscopy and high-temperature superconductivity, has acquired an outstanding reputation in basic physics research. Principal interests in physics and laser science are novel microscopies to the nanometre scale, high-Tc superconductors, numerically intensive computation,

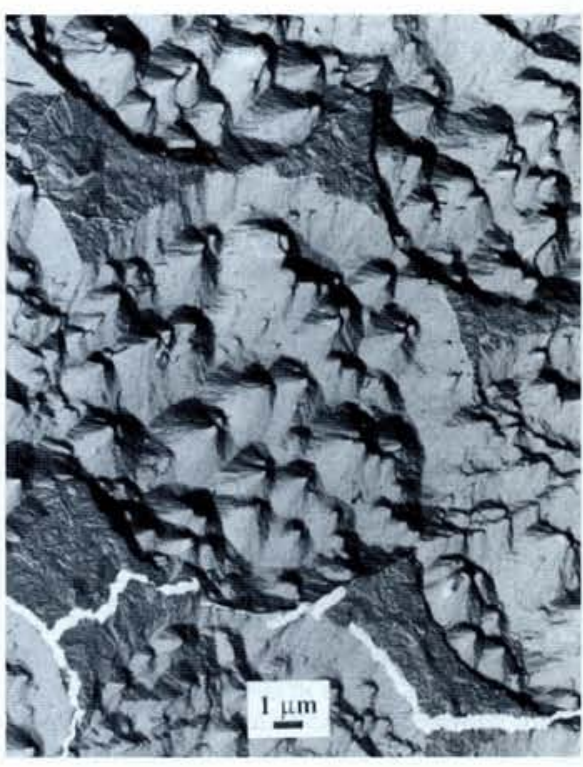

A photomicrograph, perpendicular to the main fibre direction, of an etched cross-section through hot-compacted polyethylene fibre.

would not be possible: but not to Professor V. Marikhin, Dr. L. Myasnikova and their colleagues. They accomplished the impossible and organized a Conference which was exceptional in every way. To attend and live in St. Petersburg in the season of the white nights was a great privilege for the twenty five western scientists who joined ninety Russian colleagues at this most pleasant and scientifically valuable occasion. The only cautionary note is that, in contrast to previous experience, very few attended from countries of the former eastern bloc: obtaining hard currency is the new barrier to attendance. This will need to be addressed to ensure that in future good science and scientists from all countries are able to attend Europhysics conferences. The next meeting is titled "Transitions in Oligomer and Polymer Systems" (Ulm, Germany; 27 September - 1 October 1993).

D.C. Bassett Chairman, EPS Macromolecular Board

ited number of "lightweight" partnerships which move quickly into a large number of niche markets, there is also a trend to form joint ventures with other companies to provide sufficient growth potential. There has been speculation in the press that a shift to applied research means that about 100 posts in basic research will go in the next few years.

Editor - One of IBM's goals was to be "famous for our science". Is this still the case? James McGroddy, your Director of Research, speaks of this science being a "vital" part of IBM. What does he mean?

Dr. Kümmerle - IBM has dual goals: it wants to be famous in science and technology and its science must also be relevant, in other words vital to IBM. The situation was established a long time ago and it is "burned in": it's part of the culture. We are famous by putting down stakes in selected fields as opposed to a "me too" strategy. The dual goal remains durable and nothing has changed in this respect. 
Why do you have this commitment? Does it make business sense?

There is a long list of reasons and no single argument. We want to be famous partly to show that we appreciate the long-term potential of certain things. It also makes good business sense to keep evaluating alternatives, preparing the ground for the future. However, there are clearly other considerations, not least the fact that being famous gives lustre. This has always been the case and it remains so today.

Roughly $15 \%$ of the Research Division's work covers fundamental topics. This is equivalent to about 90 M\$US or $0.13 \%$ of annual turn-over. It seems to be very little for a company based on electronic hardware. Do you think the level is sufficient?

We see the situation differently in the Research Division. We don't see applied and basic research as being different. We don't have a notion of $x$ percent of business spent on such and such. I shall give an analogy: imagine a food chain. At the top we have customer solutions built on tools, systems, manufacturing operations, and scientific knowledge as we move down the chain. Traditionally we have focussed on the lower levels; the trend now is to focus on the top few levels. This means we are looking at the bottom levels to see what should be done with them. The reason for the trend is that it is increasingly more difficult to differentiate solutions at lower levels and be unique. So our R. \& D. will move higher up the food chain: but we shall not abandon lower levels. There will be a shift and we are making an evaluation of what should be done - of where to put down stakes. We have always done this, of course, but it is becoming more important as we face today's changes.

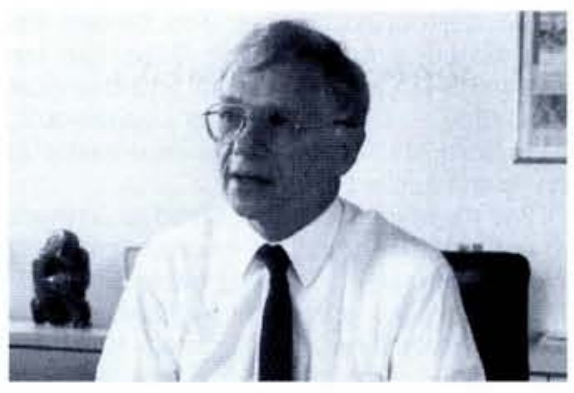

\section{IBM's Karl Kümmerle}

There is an increasing tendency in basic research for companies to collaborate with public institutions (e.g., within EC programmes, your long-standing collaboration with the ETH in computer science, etc.). Does this mean that it is now less important to control exceptional strategic breakthroughs because their exploitation is beyond a single company's capabilities ?

Zurich is the European arm of the Research Division and we have played an important rôle characterised by intensive interaction and collaborative projects. A new feature is increasing collaboration with European companies. Why are we doing this? For a variety of reasons including to be exposed to new approaches, especially those coming from top-notch European talent. It is also beneficial to us we if we can help shape research projects at universities.

Has our attitude has changed because we can no longer control breakthroughs? There is no change here. IBM will be famous; we shall stay in fundamental research; we are always thinking of the future in close collaboration with the European academic community. But there may be a shift in emphasis.
There are indications that the Division will emphasize new rôles such as helping to reduce "cultural barriers" separating say research and product development; responding directly to customers technical needs; having staff from outside research work within the Division (Dr. McGroddy speaks of "entanglement"). Will these new tasks make it more difficult to pursue and exploit basic research?

I have a hard time answering this question as we do not differentiate basic and applied research. Maybe physics can draw a line but we don't as we focus higher up the food chain. Differentiating means deciding where to put stakes in the ground. We also have a broad spectrum of customers ranging from end users, lines of business, manufacturing sites, and new entities with different needs. The tendency is for the spectrum to widen and this will give more opportunities.

\section{Could you expand a little?}

We used to be well prepared for "driving" change. It is now up to us to be explorers to look at new scenarios, so in this sense there is a broader spectrum.

You distinguish between basic research to generate scientific knowledge and applied research to extend or improve existing technologies. You say they are complementary and mutually beneficial. Can you explain why?

The distinction is irrelevant: we don't go into great efforts to distinguish. If you look at the discovery of the STM, the idea was not to do basic research. What we had were problems with Josephson junction technology (specifically, surface oxidation): a physicist had the idea of approaching this with a local method and to contribute to practica applied technology. The vision was that
The Foundation for Fundamental Research on Matter

The foundation FOM is an organisation for research in the aned of physics with some 1700 entoloyees Research is executed by task-forces at untwersity laboratories and institutes. The National institute for Nuclear Phusies and tigh Energy Phusics (NKHHF) in Amsterdam is one of these institutes, 19 coopreration of FOM, the Free Utiwersity (Vt) in Amsterdam, the liniwersity of Amsterdan (UvA) and Cathotic University of Nimiegen (KUN) The NIKHEF staff counts about 350 people spread ower two sections. Afost experiments of the Nuclear Plitsics section (K) use their own electron accelerator MEA. For the experimentat program of the fitgt Energy Physics section (H) the focitities of CERN and Desy are used.

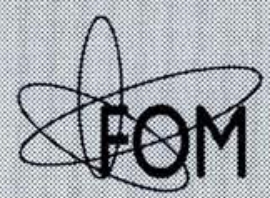

\section{Experimental physicists $\mathrm{m} / \mathrm{f}$}

Applications are invited for postdoctoral positions in (high-energy) nuclear

physics research at the section Nuclear Physics of the National Institute for Nuclear Physics and High-Energy Physics (NIKHEF) at Amsterdam. The physics staff consists of some 50 positions, half of which are for graduate students and postdoctoral fellows. The Institute avails of well equiped mechanical and electronic workshops and has modern computing facilities. There is a strong theory group that has its own research programme and provides support in the interpretation of experimental results.

Experimental research is performed with high-intensy beams of up to 900 $\mathrm{MeV}$ electrons from the pulse stretcher/ storage ring AmPS at NIKHEF.

The Institute participates in the Spin Muon Collaboration at CERN. A challenging new direction of research in development concern experiments with polarized internal targets and polarized electrons, both at AmPS and at HERA (DESY, Hamburg). Internal target physics requires expertise in e.g. high power lasers, polarization techniques and accelorator physics.

\section{Requirements}

Candidates should have a $\mathrm{PhD}$ degree preferably in experimental nuclear and/or elementary particle physics. Both junior candidates and candidates with relevant postdoctoral research experience are invited to apply.

\section{Information}

Further information may be obtained from the Scientific Director, Prof. P.K.A. de Witt Huberts, telephone: +31-205922163, telefax: +31-205922165 e-mail:

marijke@paramount.nikhefk.nikhef.nl.

\section{Applications}

Letters of application, including curriculum vitae, list of publications and the names of at least three references are to be sent within three weeks after the publication of the advertisement to the personnel officer mr. T. van Egdom, P.O. Box 41882, 1009 DB Amsterdam, the Netherlands.

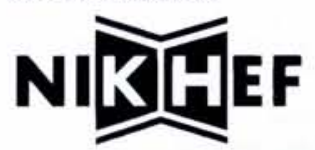




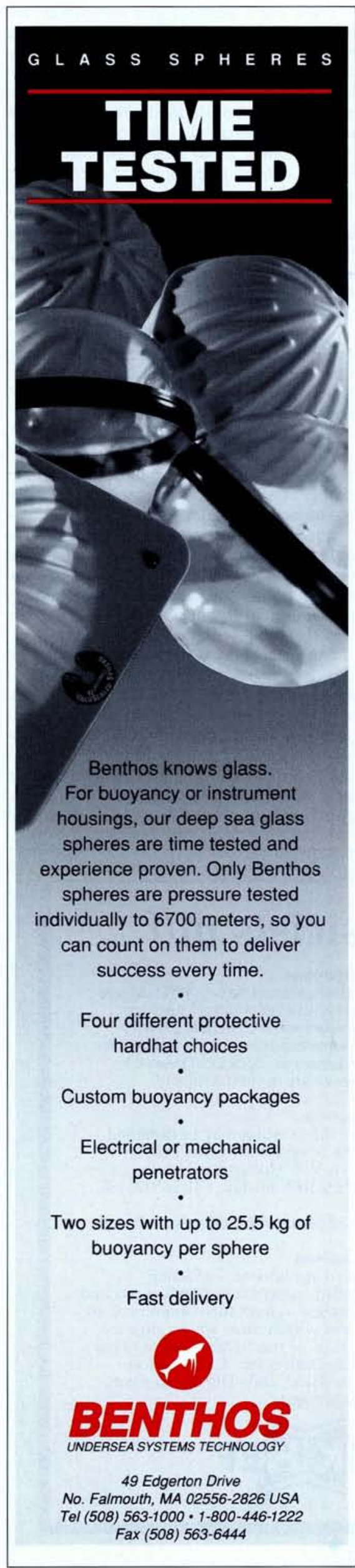

there were opportunities. The project got redirected, leading to the STM. But we needed a close commitment and technical efficiency - a "continuum of capabilities". This also shows the complementarity of basic and applied research.

Will the shift in emphasis lead to changes in the way IBM organizes its basic research? How are the changes being felt in Zurich? In particular, the company announced this week a further reduction in staff. Will there be changes in Zurich?

Zurich is currently evaluating areas to look for opportunities for "the world of IBM". This is something we have always done and we

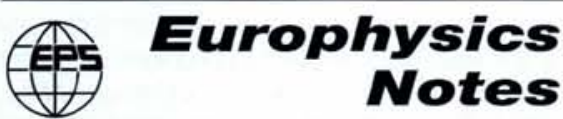

\section{- New Elements Named}

A team from the Gesellschaft für Schwerionenforschung (GSI), Darmstadt, Germany, led by Professors Peter Armbruster and Gottfried Münzenberg has been attributed with the discovery in 1981-4 of three new elements by a special IUPAP/IUPAC commission. The names of the new elements, made public at a ceremony at GSI on 7 September, are Nielsbohrium (element 107); Hassium (108) after the State of Hasse, GSI's co-founder; and Meitnerium (109) after the nuclear physicist Lise Meitner.

\section{- New CERN Director}

CERN's Committee of Council proposed last month Professor C.H. Llewellyn Smith as CERN's next Director General to replace Professor Carlo Rubbia. Endorsement by Council in December would mean his taking up duties on 1 January 1994. Professor Llewellyn Smith, who will be 50 next month, heads the University of Oxford Physics Department and is Chairman of the CERN Scientific Policy Committee. Educated at Oxford, he has been closely involved with CERN since 1968 when he was a Fellow in the Theoretical Studies Division.

\section{SSC Funding Maintained}

A US Senate vote in August on the fiscal 1993 Water and Energy Appropriations Bill granted $550 \mathrm{M} \$$ in Department of Energy funding for the 8200 MS SSC collider under construction in Texas. The difference with the House of Congress vote on a 17 June amendment to reduce funding to $34 \mathrm{M} \$$ to effectively halt the project was resolved by a House-Senate Conference Committee that agreed on $484 \mathrm{MS}$. The final $247 / 143$ vote by the House on 17 September on a compromise bill accorded $515 \mathrm{M} \$$, some $100 \mathrm{M}$ \$ less than was originally sought.

\section{- Partnerships No Match for Programmes}

The US President appointed on 1 September the first Director of the National Coordinating Office for the $800 \mathrm{MS}$, 5-year High Performance Computing and Communications (HPCC) programme. HPCC has ambitious plans to develop a digital National Research \& Education Network with Gbit/s capacity and computers with scalable performance up to a teraflop (10 12 operations/s). Lattice quantum chromodynamic (QCD) calculations in high-energy physics are often considered as an "ice-breaker" for the later. Hence, Columbia University which maintain the dual goal. With respect to the physical sciences, I don't see a fundamental change, but there might be a slight shrinking.

Is there a possibility of a RCA situation? (Editor's note: when GE in the USA bought RCA, the RCA lab in Zurich was absorbed into the Paul Scherrer Institute which was seeking at the time to extend activities).

There is no indication of this. Zurich, I believe, will remain one of the Division's three core labs. I don't see physics being squeezed out: activities in the area are important. They may be different in size and scope as we shift emphasis higher up the food chain.

built a 16 Gflops (peak speed) QCD machine joined two other universities to form one cornerstone of the US teraflop initiative - a 40 M\$ project for a 2.5 Tflops massively paralle computer that would run QCD calculations by 1995 using technology related to Thinking Machine Corp.'s CM-5 machine. Professor Enzo Marinari from Italy's INFN in Rome, in reviewing QCD calculations at the CHEP92 conference in Annecy, France, felt the schedule was "impressive but very tight" and the approach cost-effective. He said the trend elsewhere is towards partnerships. The INFN that stared running QCD calculations 4 years ago using a purpose-built 1 Gflops machine has developed the APE series of massively parallel machines. It recently signed an agreement with Laben, to produce and sell from mid-1993 a 96 Gflops APE-100 mode comprising a $4 \times 4$ array of the APE 6 Gflops model commissioned 6 months ago.

Overall, however, European-level initiatives in HPCC's two main areas remain low key: the follow-up of a 1991 study for the Commission of the EC of high-performance computing [EN 22 (1991) 64] chaired by Professor C. Rubbia has been released as we go to press. It defines and costs severa options for consideration in the EC's next 5 . year budget presently being discussed by Ministers. Meanwhile, in a parallel initiative, a Consultative Forum of specialists has issued a working paper called Challenges Ahead that recommends spending about $200 \mathrm{MECU}$ for high-capacity academic and research networks.

\section{Mobility Scheme Admits $\mathbf{1 1 5}$}

The first meeting in Geneva on 9/10 October of the Mobility Committee of the European Mobility Scheme for Physics Students decided that the Universities of Ghent and Hannover would coordinate EC funding applications. 115 Institutions were admitted to the scheme wich starts next autumn.

\section{- Trento Recommended for Nuclear} Theory Centre

The Steering Commitee for a future European Center for Theoretical Nuclear Physics meeting in Orsay on October 12 to consider site proposals from Legnaro and the University of Trento, Italy, decided to recommend the latter to NUPECC as it afforded the "best opportunity". Recommended for the initial Board of Directors are J.-P. Blazlot (Saclay), S. Fantoni (Trieste), B. Mottelson (Copenhagen), C. Pethick, and W. Welse (Regensburg). Proposal from Copenhagen and Utrecht had been withdrawn earlier owing to poor financial possibilities. 